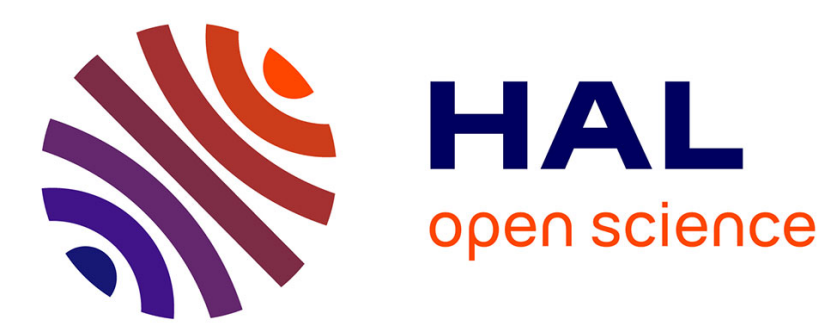

\title{
Slavoj Zizek, Fabien Tarby, A travers le réel. Entretiens avec Fabien Tarby
}

Pascal Fugier

\section{To cite this version:}

Pascal Fugier. Slavoj Zizek, Fabien Tarby, A travers le réel. Entretiens avec Fabien Tarby . 2011. hal-01672890

\section{HAL Id: hal-01672890 \\ https://hal.science/hal-01672890}

Submitted on 27 Dec 2017

HAL is a multi-disciplinary open access archive for the deposit and dissemination of scientific research documents, whether they are published or not. The documents may come from teaching and research institutions in France or abroad, or from public or private research centers.
L'archive ouverte pluridisciplinaire HAL, est destinée au dépôt et à la diffusion de documents scientifiques de niveau recherche, publiés ou non, émanant des établissements d'enseignement et de recherche français ou étrangers, des laboratoires publics ou privés. 
Pascal Fugier, «Slavoj Zizek, Fabien Tarby, A travers le réel. Entretiens avec Fabien Tarby », Lectures [En ligne], Les comptes rendus, 2011, mis en ligne le 31 mars 2011. URL: http://journals.openedition.org/lectures/1333

1Dans ce livre d'entretiens, Slavoj Žižek explicite et articule les éléments clefs de sa pensée. Pensée que nous pouvons qualifier d'atypique du fait qu'elle se traduit par une lecture philosophique de la psychanalyse et réciproquement une lecture psychanalytique de la philosophie. De même, si l'auteur emprunte une posture critique et n'hésite pas à donner une signification politique à sa réflexion, tournée vers l'analyse des «coordonnées idéologiques d'aujourd'hui » (p. 182), l'originalité de sa philosophie transparaît aussi sur le plan méthodologique. Slavoj Žižek illustre ainsi ses problématiques philosophique et psychanalytique en prenant comme "terrains" le cinéma et la musique, analysant aussi bien une superproduction américaine comme Avatar que les films réalisés par David Lynch, Alfred Hitchcock ou encore le hard-rock du groupe allemand Rammstein (chapitres IX et $\mathrm{X}$ ). Et il faut reconnaître ici la dextérité avec laquelle Fabien Tarby discute cette pensée critique et lui apporte un éclairage singulier, en opérant notamment une lecture croisée entre Slavoj Žižek et la philosophie d'Alain Badiou (dont Fabien Tarby est un spécialiste). Par ailleurs, les différents chapitres de ce livre d'entretiens, eux-mêmes subdivisés en différents thèmes, offrent un tour d'horizon de cette philosophie mais sans sacrifier sa complexité et les multiples mouvements et déplacements qui caractérisent la pensée de Slavoj Žižek, à l'image de la manière dont il peut tantôt adopter, tantôt critiquer la psychanalyse de Jacques Lacan. Ce livre d'entretiens constitue donc une bonne introduction à I'œuvre de Slavoj Žižek ${ }^{1}$ et nous pouvons désormais revenir sur certains de ses éléments clefs.

2Si les premières paroles retranscrites du philosophe nous ont quelque peu désarçonné, lorsqu'il érige une frontière bien intellectualiste entre sa trajectoire biographique et «la vérité » de sa théorie (p. 9), le premier chapitre annonce toute la qualité des échanges : après avoir discuté le concept de soustraction chez Alain Badiou (p. 11), Slavoj Žižek distingue très clairement les approches freudienne et jungienne du symbolique, Sigmund Freud contextualisant la signification des symboles mobilisés par les individus tandis que Carl Gustave Jung, fort de son mysticisme, les universalise (p. 12-15). Mais Slavoj Žižek est tout aussi critique vis-à-vis de la tentation positiviste de la psychanalyse. Et il recourt alors à la critique hégélienne de «l'universalité abstraite » (p. 15) pour dénoncer les psychanalystes qui ont toujours le dernier mot sur leurs analysants, pressés

\footnotetext{
${ }^{1}$ Nous pouvons aussi mentionner à ce propos l'ouvrage collectif dirigé par Raoul Moati,
} Autour de Slavoj Žižek. Psychanalyse, marxisme, idéalisme allemand (Paris, PUF, 2010). 
qu'ils sont de repérer par exemple chez eux les traits typiques du névrosé (p. 16). S'interrogeant avec Jacques-Alain Miller sur les conditions sociales de possibilité de la psychanalyse, il défend la légitimité et la portée heuristique d'un usage non clinicien de la psychanalyse, réaffirmant que « la psychanalyse, ce n'est pas seulement le divan. La psychanalyse est une pensée » (p. 19). Ce qui le conduit à mettre en valeur par la même occasion le compromis qu'il tente de réaliser « entre les deleuziens et les lacaniens » (p. 17).

3Le second chapitre est le plus psychanalytique de l'ouvrage. Intitulé «I'objet "Lacan" », Slavoj Žižek y introduit notamment I'originalité de sa thèse concernant la triade conceptuelle nouant le réel, le symbolique et l'imaginaire. Selon lui, cette triade « se répète » dans le réel. Il conçoit ainsi un « réel imaginaire » (prenant la forme du monstre, de l'horreur, du horla), un « réel symbolique » (qui renvoie au non-sens et qu'illustre la mécanique quantique) et un «réel réel » (qui est purement formel, c'est un « obstacle », une « différence mais absolument sans distance »). À partir de cette thèse, Slavoj Žižek se positionne vis-à-vis des lecteurs et représentants contemporains de Jacques Lacan. À commencer par JacquesAlain Miller, dont il reconnaît l'héritage et les qualités pédagogiques, tout en regrettant sa lecture «téléologique » de Jacques Lacan (p. 34). De même s'oppose-t-il à « la lecture traditionnelle des figures de la sexuation de Lacan » (p. 40), préférant, pour reprendre la formule de Fabien Tarby, « dialectiser les formules de Lacan sur la masculin et le féminin » (p. 41).

4Si le second chapitre est le plus lacanien, le troisième est incontestablement le plus hégélien, Fabien Tarby incitant Slavoj Žižek à développer sa lecture peu « conventionnelle » du philosophe. C'est alors un Hegel penseur de la contingence qui est mis en avant. Et s'il souligne son historicisme, il tient à « ébranler cette image de Hegel, l'idéaliste absolu, la clôture, le système » ( $p .56)$, en nouant notamment la négativité hégélienne avec la pulsion de mort feudienne (p. 64).

5Les chapitres suivants réservent davantage de place à la mise en perspective de la réflexion de Slavoj Žižek par des philosophies plus contemporaines, comme celles d'Alain Badiou (chapitre IV), Gilles Deleuze et Jacques Derrida (chapitre VI). Ayant aussi effectué un livre d'entretiens avec Alain Badiou ${ }^{2}$, Fabien Tarby invite le philosophe slovène à explorer les problématiques de l'événement, du simulacre et du sujet. Or, en prolongeant la thèse de Guy Debord sur la « société du spectacle », les auteurs abordent un aspect déterminant de l'idéologie dominante, que Slavoj Žižek qualifie de «cynisme éclairé » (p. 99). Il définit aussi cet aspect idéologique comme «l'énigme d'un savoir inefficace » (p. 100) et qui tient au fait que les explications (philosophique, psychanalytique, sociologique...) exercent désormais moins d'effets de vérité. Les individus ne peuvent plus être considérés comme les victimes inconscientes de la société du spectacle. Ils savent qu'ils sont manipulés par le spectacle. Cela

${ }^{2}$ A. Badiou et F. Tarby, La philosophie et l'événement, Paris, Germina, 2010. 
dit, si « nous ne sommes pas fascinés, cependant le spectacle marche » (p. 100). Se référant à l'analyse de Luc Boltanski et Ėve Chiapello sur Le nouvel esprit du capitalisme ${ }^{3}$, Slavoj Žižek voit dans cette énigme le signe de la réappropriation du message de 68 par le capitalisme.

6Dans le chapitre V, Fabien Tarby interroge Slavoj Žižek sur ses analyses des mythologies et des idéologies. Žižek reprend notamment la thèse marxienne du fétichisme de la marchandise et l'associe avec la problématique lacanienne de la logique symbolique du «grand Autre » et sa thèse selon laquelle «Dieu est inconscient». Ce qui le permet de soutenir que les individus contemporains ne sont athées qu'« en apparence » (p. 115).

7Enfin, les chapitres VII et VIII abordent l'engagement politique du philosophe, à commencer par sa présentation aux élections présidentielles slovènes de 1991. Mais les auteurs maintiennent pour autant un vocable analytique, Slavoj Žižek affirmant par exemple que «nous avons besoin d'un communisme sans Grand Autre » (p. 144). Et quand il s'agit de se positionner par rapport à certaines figures intellectuelles françaises, Slavoj Žižek mentionne notamment les efforts de Bernard-Henry Levy afin de « dépolitiser mai 1968 » (p. 153). Il s'en démarque bien entendu, de même qu'il prend ses distances vis-à-vis du conservatisme de Pierre Legendre.

80n l'aura compris, il ne faut pas attendre de cet ouvrage une synthèse de la philosophie de Slavoj Žižek et des dizaines d'ouvrages que ce dernier publie depuis désormais près de trente ans. Par contre, nous y retrouvons toute l'effervescence d'une pensée qui n'hésite pas à nouer et à confronter des disciplines et des auteurs a priori discordants voire totalement incompatibles, comme Gilles Deleuze, Jacques Lacan et Karl Marx. Et c'est tout le mérite de Fabien Tarby que d'avoir conduit Slavoj Žižek à livrer plusieurs éléments déterminants d'un échafaudage théorique qui se présente comme une véritable «totalité en marche $»^{4}$.

\footnotetext{
${ }^{3}$ Paris, Gallimard, 1999.

${ }^{4}$ Nous nous référons ici à la sociologie de la connaissance de Georges Gurvitch et à sa conception sartrienne de la dialectique : "Sartre insiste avec beaucoup de force sur le fait que les totalités dont il s'agit dans la dialectique ne sont jamais des totalités toutes faites, statiques, mais des totalités en train de se faire ou de se refaire, totalités dynamiques, totalités mouvantes, "totalités en marche", selon notre propre terminologie » (G. Gurvitch, Dialectique et sociologie, Paris, Flammarion, 1977, p. 204).
} 